\title{
HIDRÓLISE E FERMENTAÇÃO SIMULTÂNEA DO BAGAÇO DE CANA-DE-AÇÚCAR PRÉ-TRATADO HIDROTERMICAMENTE
}

\author{
G. M. SILVA ${ }^{1}$ e A. J. G. CRUZ ${ }^{1,2}$ \\ ${ }^{1}$ Programa de Pós-Graduação em Engenharia Química, Universidade Federal de São Carlos \\ ${ }^{2}$ Departamento de Engenharia Química, Universidade Federal de São Carlos \\ E-mail para contato: gislenemota@ hotmail.com
}

RESUMO - O objetivo deste trabalho foi realizar o pré-tratamento hidrotérmico do bagaço de cana-de-açúcar (BCA), e em seguida, realizar ensaios de Sacarificação e Fermentação Simultâneas (SSF), utilizando levedura liofilizada Saccharomyces cerevisiae. O BCA foi tratado em três condições: $195{ }^{\circ} \mathrm{C} / 10 \mathrm{~min}, 195{ }^{\circ} \mathrm{C} / 10 \mathrm{~min}$ com $0,021 \% \mathrm{NaOH}$ e $195{ }^{\circ} \mathrm{C} / 10 \mathrm{~min}$ com $0,5 \% \mathrm{NaOH}$. Depois da etapa de pré-tratamento a fração insolúvel foi separada da fração líquida por filtração e lavada com água para a remoção dos açúcares solúveis e compostos inibidores. As frações sólidas foram analisadas quimicamente. Após as caracterizações, os sólidos foram hidrolisados utilizando celulase comercial e fermentados. As SSFs foram realizadas em shaker com 10 e $15 \%(\mathrm{~m} / \mathrm{m})$ de carga de sólidos a $250 \mathrm{rpm}$ a $37{ }^{\circ} \mathrm{C}$ por $120 \mathrm{~h}$. Os resultados mostraram que os BCAs tratados apresentaram satisfatória remoção de hemicelulose. Nas SSFs obteve-se máxima conversão em glicose de 47,7 \% (ensaio com $15 \%$ de sólidos sem deslignificação) e rendimento em etanol de 41,8\% (ensaio com $10 \%$ de sólidos com deslignificação alcalina).

\section{INTRODUÇÃO}

Os materiais lignocelulósicos são muitos abundantes no Brasil e podem ser obtidos de vários processos agroindustriais, tais como na produção de açúcar e etanol nas usinas. Estes resíduos agroindustriais contêm grandes quantidades de açúcares fermentescíveis que podem ser hidrolisados e fermentados para a produção de etanol de segunda geração (2G) (Silva, 2011).

O bagaço de cana-de-açúcar (BCA) é constituído basicamente de celulose, hemicelulose e lignina. A celulose é um polímero linear do dímero glicose-glicose (celobiose), rígido e difícil de ser hidrolisada. A sua hidrólise completa gera a glicose (açúcar de seis carbonos fermentescível). A hemicelulose é um heteropolímero constituída principalmente do monômero xilose e com várias ramificações de manose, arabinose, galactose entre outras. A sua hidrólise é mais fácil que a da celulose, porém a fermentação da xilose (açúcar de cinco carbonos) é um pouco mais difícil. Por fỉm, a lignina é uma macromolécula aromática heterogênea formada por ligações éter biologicamente estáveis. Sua estrutura química é hidrofóbica e complexa. É formada por unidades de fenilpropano derivadas da polimerização oxidativa de três alcoóis precursores: trans-p-cumárico, trans conífero e trans sinapílico (Curvelo, 1992).

Várias tecnologias estão disponíveis para a conversão da biomassa em açúcares monoméricos 


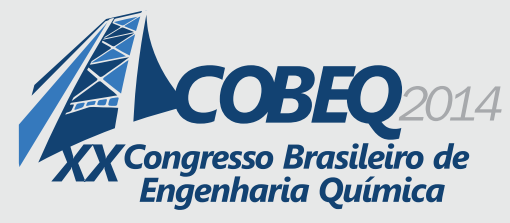

19 a 22 de outubro de 2014
Florianópolis/SC

(Santos et al., 2011). O diferencial entre as tecnologias está no catalisador utilizado na etapa de hidrólise dos polissacarídeos do material lignocelulósico (Kádár et al., 2004).

As etapas de hidrólise enzimática e fermentação podem ser conduzidas simultaneamente, processo que é conhecido como Sacarificação e Fermentação Simultâneas (SSF) (Öhgren et al., 2006). A SSF é um processo mais eficiente que a Sacarificação e Fermentação Separadas (SHF), pois reduz o acúmulo de açúcares que podem inibir a enzima durante a etapa de hidrólise (Brethauer e Wyman, 2010). Além disso, a combinação das etapas de hidrólise e fermentação diminui os custos do processo e reduz os riscos de contaminação (Olofsson, Bertilsson e Gunnar, 2008).

Este trabalho avaliou a etapa de pré-tratamento hidrotérmico do BCA e realizou experimentos na configuração Sacarificação e Fermentação Simultâneas.

\subsection{Pré-tratamento hidrotérmico}

O pré-tratamento hidrotérmico tem atraído grande atenção pelo fato de usar apenas água como solvente. No pré-tratamento hidrotérmico, dependendo da condição operacional empregada (temperatura e tempo de reação), é possível obter significativa remoção da hemicelulose com retenção da celulose na biomassa e melhoramento da sua digestibilidade pela celulase (Hu et al., 2011).

A água líquida abaixo do ponto crítico é favorecida para realizações de reações químicas. A constante de ionização da água aumenta com a temperatura e é cerca de três ordens de grandeza maior do que a temperatura ambiente. Além disso, a constante dielétrica da água diminui com a temperatura. A baixa constante dielétrica da água permite que a água líquida dissolva os compostos orgânicos, enquanto que uma alta constante de ionização da água promove em um meio ácido a hidrólise da biomassa, através da clivagem das ligações de éter e éster favorecendo a hidrólise da hemicelulose (Savage, 1999; Kumar e Gupta 2009 e Franck, 1998). O ácido acético formado a partir da desacetilação parcial da fração hemicelulósica atuará como catalisador da reação da hidrólise da biomassa promovendo a despolimerização da hemicelulose (processo autocatalítico).

O pré-tratamento hidrotérmico é tipicamente conduzido em temperaturas entre 150 a $220{ }^{\circ} \mathrm{C}$. A depender das condições operacionais ocorre uma maior ou menor despolimerização da hemicelulose. Esse fato pode levar a maior concentração de oligossacarídeos e/ou do monômero (xilose). A recuperação desse monômero pode variar entre 88 a 98 \% (Kumar et al., 2011).

Valores de pH abaixo de 3 podem causar a precipitação da lignina e também catalisar a degradação da xilose. Para evitar a formação de inibidores, o pH deve ser controlado entre 4 e 7 durante a etapa de pré-tratamento. Nesta faixa de $\mathrm{pH}$ minimiza-se a formação de monossacarídeos e, por conseguinte, a formação de produtos de degradação. Manter o pH perto do neutro (5 a 7) evita a formação de inibidores da fermentação durante a etapa de pré-tratamento (Bobleter 1994; Hendriks e Zeeman 2009).

A intensidade do pré-tratamento hidrotérmico pode ser quantificada por um parâmetro denominado fator de severidade $\left(\mathrm{r}_{\mathrm{o}}\right)$. Este parâmetro foi proposto por Overend e Chornet em 1987 (Equação 1). 


$$
\log \left(\mathrm{r}_{\mathrm{o}}\right)=t \cdot \exp \left(\frac{T-100}{14,75}\right)
$$

onde: $t$ é o tempo de residência (em minutos) e T é a temperatura $\left(\mathrm{em}^{\circ} \mathrm{C}\right)$.

Este fator pode ser usado para medir o efeito combinado de ambas as variáveis, tempo e temperatura, em um dado pré-tratamento.

\section{MATERIAIS E MÉTODOS}

\subsection{Bagaço de cana-de-açúcar (BCA)}

O bagaço de cana-de-açúcar utilizado neste projeto foi cedido pelo CTC (Centro de Tecnologia Canavieira, Piracicaba, SP). Neste trabalho as amostras de BCA foram peneiradas em uma malha de $3 \mathrm{~mm}$.

\subsection{Pré-tratamento do BCA}

O BCA in natura foi pré-tratado hidrotermicamente a $195{ }^{\circ} \mathrm{C} / 10 \mathrm{~min}$ com agitação de $200 \mathrm{rpm}$ na proporção $1: 10(\mathrm{~m} / \mathrm{m})$. O material após o primeiro pré-tratamento foi filtrado e em seguida, desliginificado com solução de hidróxido de sódio $(\mathrm{NaOH})$. As concentrações das soluções foram $0,021 \%$ e $0,5 \%$ de $\mathrm{NaOH}$. O material tratado hidrotermicamente foi desliginificado na proporção 1:10 (m/v) em autoclave a $121{ }^{\circ} \mathrm{C}$ por $30 \mathrm{~min}$. Após o tempo de reação, o BCA desliginificado com $0,021 \%$ de $\mathrm{NaOH}$ foi lavado três vezes com volume de $500 \mathrm{~mL}$ de água deionizada $\left( \pm 60{ }^{\circ} \mathrm{C}\right) . \mathrm{O}$ BCA tratado com 0,5\% de $\mathrm{NaOH}$ foi lavado até $\mathrm{pH} 7,0$. Determinou-se a umidade dos BCAs deslignificados. As amostras foram armazenadas em sacos plásticos herméticos em câmara fria para posterior caracterização química (metodologia descrita por Gouveia et al., 2009) e hidrólise enzimática.

\subsection{Sacarificação e Fermentação Simultâneas (SSF)}

Ensaios de SSFs foram realizados com o material tratado a $195{ }^{\circ} \mathrm{C} / 10 \mathrm{~min}$ seguido de deslignificação com $0,021 \%$ e $0,5 \%$ de solução de $\mathrm{NaOH}$. Todos os ensaios foram realizados em triplicatas.

O inóculo foi preparado em frascos de Erlenmeyer de $250 \mathrm{~mL}$ em shaker $32{ }^{\circ} \mathrm{C}$ com agitação de $200 \mathrm{rpm}$ por 16 horas. O inóculo foi constituído de 5,0 g/L de extrato de levedura, 10,0 g/L de peptona, 25,0 g/L de glicose e 2,0 g/L de Saccharomyces cerevisiae (liofilizada PE-2). Terminado o tempo de reação, o meio foi centrifugado em tubos Falcon de $45 \mathrm{~mL}$ a $6.000 \mathrm{rpm}$ por $10 \mathrm{~min}$. Retirouse o sobrenadante e suspendeu-se o inóculo com tampão citrato $50 \mathrm{mM}, \mathrm{pH} 4,8$. Adicionou-se em 
cada frasco 0,5 mL de inóculo com densidade ótica (DO) de 0,5. A DO foi calculada segunda Equação 2 (NREL, 2008).

Volume total de inóculo $(\mathrm{mL})=\left(\frac{\text { volume de hidrolisad o }(\mathrm{mL}) \times 0,5}{\text { D.O final do inóculo }}\right) x($ número de frascos hidrolisad os +1$)$

O meio de fermentação foi preparado em um balão volumétrico de $250 \mathrm{~mL}$. O meio foi constituído de 5,0 g/L extrato de levedura, 3,0 g/L de peptona, 2,5 g/L de sulfato de magnésio heptahidratado $\left(\mathrm{MgSO}_{4} .7 \mathrm{H}_{2} \mathrm{O}\right), 1,0 \mathrm{~g} / \mathrm{L}$ de fosfato de dipotássio $\left(\mathrm{K}_{2} \mathrm{HPO}_{4}\right)$ e 5,0 $\mathrm{g} / \mathrm{L}$ de sulfato de amônio $\left(\mathrm{NH}_{4}\right)_{2} \mathrm{SO}_{4}$.

As SSFs foram realizadas em frascos de Erlenmeyer de $250 \mathrm{~mL}$ em shaker a $250 \mathrm{rpm}$ a $37{ }^{\circ} \mathrm{C}$ por $120 \mathrm{~h}$ na proporção de 10 e $15 \%$ de carga de sólidos e carga enzimática de 20 FPU (Accellerase 1500)/g BCA tratado. Nos ensaios com $15 \%$ de sólidos adicionou-se esferas metálicas para ajudar na dissolução do BCA tratado. Adicionou-se 5,0 mL de meio de fermentação, 2,5 mL de tampão citrato $\mathrm{pH} 4,8(50 \mathrm{mM}), 0,5 \mathrm{~mL}$ de inóculo de $\mathrm{DO}$ de 0,5 e água deionizada até massa total de 50,0 g. Alíquotas foram retiradas e filtradas em membrana de celulose $0,2 \mu \mathrm{m}$ para análise por Cromatografia Líquida de Alta Eficiência (CLAE).

\section{RESULTADOS E DISCUSSÃO}

A Tabela 1 apresenta os resultados dos rendimentos mássicos (R) obtidos após cada etapa de pré-tratamento. A perda de massa nas amostras que foram deslignificadas em relação à amostra que somente sofreu tratamento hidrotérmico foi de $8,7 \%$ para o BCA hidrotérmico $/ 0,021 \% \mathrm{NaOH}$ e $15,6 \%$ para o BCA tratado hidrotermicamente $/ 0,5 \% \mathrm{NaOH}$.

Tabela 1. Rendimentos mássicos $(\mathrm{R})$ obtidos na etapa de pré-tratamento.

\begin{tabular}{|l|c|}
\hline Condições de pré-tratamentos & $\mathrm{R}(\%)$ \\
\hline $195^{\circ} \mathrm{C} / 10 \mathrm{~min}$ & 63,4 \\
\hline $195^{\circ} \mathrm{C} / 10 \mathrm{~min} / 0,021 \%$ de $\mathrm{NaOH}$ & 57,9 \\
\hline $195^{\circ} \mathrm{C} / 10 \mathrm{~min} / 0,5 \%$ de $\mathrm{NaOH}$ & 53,5 \\
\hline
\end{tabular}

$$
\mathrm{R}=\text { massa final }(\mathrm{g}) / \text { massa inicial }(\mathrm{g})
$$

A Tabela 2 apresenta os resultados obtidos nas caracterizações químicas das amostras de BCA sem deslignificação e com deslignificação de 0,021 e 0,5 \% de solução de $\mathrm{NaOH}$. 
Tabela 2. Caracterização química das amostras de BCA (in natura e pré-tratadas).

\begin{tabular}{|l|c|c|c|c|}
\hline \multicolumn{1}{|c|}{$\begin{array}{c}\text { Componentes } \\
(\%)\end{array}$} & In natura & $195^{\circ} \mathrm{C} / 10 \mathrm{~min}$ & $\begin{array}{c}195^{\circ} \mathrm{C} / 10 \mathrm{~min} \\
\mathrm{com} 0,021 \%\end{array}$ & $\begin{array}{c}195{ }^{\circ} \mathrm{C} / 10 \mathrm{~min} \\
\mathrm{com} 0,5 \% \\
\mathrm{NaOH}\end{array}$ \\
\hline Celulose & & & $\mathrm{NaOH}$ \\
Hemicelulose & $24,5 \pm 0,8$ & $6,3 \pm 0,1$ & $3,6 \pm 0,5$ & $6,3 \pm 1,0$ \\
Lignina solúvel & $5,5 \pm 1,3$ & $2,9 \pm 0,1$ & $2,5 \pm 0,2$ & $5,2 \pm 0,7$ \\
Lignina insolúvel & $17,7 \pm 1,5$ & $27,4 \pm 2,2$ & $26,7 \pm 0,5$ & $17,9 \pm 0,2$ \\
Cinzas & $5,4 \pm 1,1$ & $4,3 \pm 0,1$ & $4,4 \pm 0,1$ & $5,1 \pm 0,2$ \\
Extrativos & $5,7 \pm 0,4$ & - & - & - \\
\hline Total & 99,6 & 96,6 & 95,9 & 96,6 \\
\hline
\end{tabular}

A partir dos resultados apresentados (Tabela 1) observa-se que o aumento da concentração de $\mathrm{NaOH}$ causa maior remoção de lignina, aumentando o teor de celulose na amostra de BCA prétratada. No entanto, o aumento na concentração de $\mathrm{NaOH}$ promove maior perda de biomassa. A remoção de hemicelulose foi satisfatória para todos os BCAs deslignificados $(83,7 \%$ para hidrotérmico, $91,4 \%$ para hidrotérmico/ $0,021 \%$ de $\mathrm{NaOH}$ e $86,1 \%$ para hidrotérmico com $0,5 \%$ de $\mathrm{NaOH})$. O teor de lignina insolúvel foi reduzido quase pela metade $(45,8 \%)$ para o BCA hidrotérmico $/ 0,5 \% \mathrm{NaOH}$. A perda de celulose foi de $16,7 \%$ e 20,6 \%, respectivamente, para BCA hidrotérmico $/ 0,021 \%$ e hidrotérmico $/ 0,5 \%$ de $\mathrm{NaOH}$.

A produção de etanol a partir da biomassa requer a conversão da celulose em glicose e, em seguida, a fermentação do açúcar a etanol (Hu et al., 2011). Neste trabalho, os ensaios de SSFs foram realizados em shaker a $37^{\circ} \mathrm{C}$ com agitação de $250 \mathrm{rpm}$ por $120 \mathrm{~h}$ com cargas de sólidos de 10 e $15 \%$ de BCA seco. A concentração de etanol foi acompanhada ao longo do tempo, como mostra a Figura 1. O melhor rendimento em etanol foi para $10 \%$ de BCA com $0,021 \%$ de NaOH cerca de $41,8 \%$ em $48 \mathrm{~h}$ de SSF. As condições de 10 e $15 \%$ de BCA sem deslignificação os rendimentos foram de 36,6 (em 96 h) e 39,5\% (em 96 h), respectivamente, Tabela 3. 


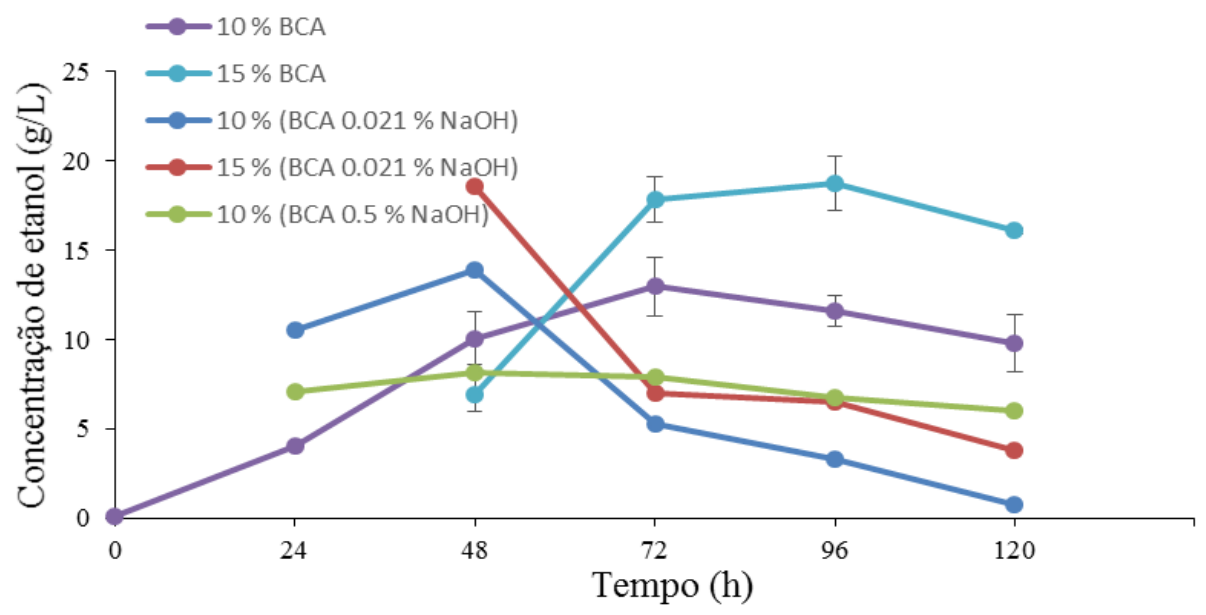

Figura 1. Gráfico da concentração de etanol ao longo dos experimentos SSFs.

Tabela 3. Parâmetros obtidos nos ensaios de SSFs.

\begin{tabular}{|l|c|c|c|c|}
\hline Condições das SSFs & $\mathrm{C}_{\mathrm{G}}(\mathrm{g} / \mathrm{L})$ & $\mathrm{C}_{\mathrm{G}(\mathrm{SSF})}(\%)$ & $\mathrm{C}_{\mathrm{E}}(\mathrm{g} / \mathrm{L})$ & $\mathrm{R}_{\mathrm{E}}(\%)$ \\
\hline $10 \%\left(195^{\circ} \mathrm{C} / 10 \mathrm{~min}\right)$ & 61,9 & 37,9 & 31,6 & 36,6 \\
\hline $15 \%\left(195^{\circ} \mathrm{C} / 10 \mathrm{~min}\right)$ & 92,9 & 47,7 & 47,5 & 39,5 \\
\hline $10 \%\left(195^{\circ} \mathrm{C} / 10 \mathrm{~min}\right.$ & 65,2 & 42,7 & 33,3 & 41,8 \\
$/ 0,021 \% \mathrm{NaOH})$ & & & 50,1 & 13,1 \\
\hline $\begin{array}{l}15 \%\left(195{ }^{\circ} \mathrm{C} / 10 \mathrm{~min}\right. \\
/ 0,021 \% \mathrm{NaOH})\end{array}$ & 98,0 & 22,0 & & 23,7 \\
\hline $\begin{array}{l}10 \%\left(195{ }^{\circ} \mathrm{C} / 10 \text { min }\right. \\
/ 0,5 \% \mathrm{NaOH})\end{array}$ & 67,3 & 24,4 & & 34,4 \\
\hline
\end{tabular}

$\mathrm{C}_{\mathrm{G}}$ : Conversão teórica total da celulose em glicose.

$\mathrm{C}_{\mathrm{G}(\mathrm{SSF})}$ : Conversão da celulose em glicose obtida na SSF.

$\mathrm{C}_{\mathrm{E}}$ : Concentração teórica total de glicose a etanol.

$\mathrm{R}_{\mathrm{E}}$ : Rendimento em etanol global (conversão teórica total da glicose em etanol).

Os resultados mostraram que a máxima conversão de celulose em glicose $(47,7 \% \mathrm{em} 48 \mathrm{~h})$ foi obtida na condição com $15 \%$ de BCA sem deslignificação. A condição com $15 \%$ de BCA deslignificado com $0,021 \%$ apresentou o menor rendimento $(22,0 \%)$. Uma possível causa seria a contaminação do BCA. Utilizando $10 \%$ de sólidos a melhor conversão foi para a condição com 0,021 $\%$ de $\mathrm{NaOH}(42,7 \%)$. 


\section{CONCLUSÃO}

Os resultados apresentados neste trabalho mostraram que o pré-tratamento hidrotérmico seguido de deslignificação com solução de $\mathrm{NaOH}$ aumentou o teor de celulose no BCA, porém diminuiu o rendimento do pré-tratamento. O teor de lignina também foi reduzido com o aumento da concentração de $\mathrm{NaOH}$ no pré-tratamento. Nos ensaios de SSFs a deslignificação não foi tão significativa, pois com $15 \%$ de BCA sem deslignificar obteve-se maior conversão em glicose $(47,7 \%)$ quando comparado com $15 \%$ de BCA deslignificado com $0,021 \%$ de $\mathrm{NaOH}(22,0 \%)$. Na produção de etanol os rendimentos para $10 \%$ BCA sem deslignificação foi de 36,6 \%. Quando o material foi deslignificado com $0,021 \%$ obteve-se uma melhora no rendimento, alcançando o valor de 41,8\%.

\section{Agradecimentos}

Ao CNPq, à CAPEs e à FAPESP pelo apoio financeiro para realização deste trabalho.

\section{REFERÊNCIAS}

BOBLETER, O. Hydrothermal degradation of polymers derived from plants. Prog. Polym Sci., v. 19, p. 797-841, 1994.

BRETHAUER, S.; WYMAN. C.; E. Rewiew: continuous hydrolysis and fermentation for cellulosic ethanol production. Bioresourc. Technol., v. 101, p. 4862-4874, 2010.

FRANCK, E. U. Fluids at high pressures and temperatures. Pure Appl. Chem., v. 59, n. 1, p. 25-34, 1987.

CURVELlO, A. A. S. Processos de deslignificação organossolve. Instituto de Química de São Carlos, USP, 1992, 110p. (Tese)

GOUVEIA, E.; R.; NASCIMENTO, R.; T.; SOUTO-MAIOR, A.; M. Validação de metodologia para a caracterização química do bagaço de cana-de-açúcar. Quím. Nov., v. 32, n. 6, p. 1500-1503, 2009.

HENDRIKS, A.; T.; W.; M.; ZEEMAN, G. Pretreatment to enhance the digestibility of lignocellulosic biomass. Bioresourc. Technol., v. 10, p 100, 2009.

HU, Z.; RAGAUSKAS, A.; J. Hydrothermal pretreatment of switchgrass. Ind. Eng. Chem., v. 50, p. 4225-4230, 2011.

KÁDÁR, Z.; SZENGYEL, Z;; RÉCZEY, K. Simultaneous Saccharification and Fermentation (SSF) of industrial wastes for the production of ethanol. Ind. Crop. Prod., v. 20, p. 103-110, 2004.

KUMAR, S.; GUPTA, R.; B. Biocrude production from switchgrass using subcritical water. Energy and Fuels, v. 23, p. 5151-9, 2009. 
LIU, C.; WYMAN, C.; E. The effect of flow rate of compressed hot water on xylan, lignin and total mass removal from corn stover. Ind. Eng. Chem. Res., V. 42, p. 5409-16, 2003.

OLOFSSON, K.; BERTILSSON, M.; GUNNAR, L. A short review on SSF - an interesting process option for ethanol production from lignocellulosic feedstocks. Biotechnol. Biofuel., 1:7, p. 1-14, 2008. ÖHGREN, K.; BENGTSSON, O.; GORWA-GRAUSLUND, M.; F.; GALBE, M.; HÄHNHÄGERDAL, B.; ZACCHI, G. Simultaneous saccharification and co-fermentation of glucose and xylose in steam-pretreated corn stover at high fiber content with Saccharomyces cerevisiae TMB3400. Scienc. Direct., v. 126, p. 488-498, 2006.

OVEREND, R. P.; CHORNET, E. Fractionation of lignocellulosic by stream-aqueous pretreatments. Philos. Trans. R. Soc. Lond., v. 321, p. 523-536, 1987.

ROGALINSKI, T.; INGRAM, T.; BRUNNER, G. Hydrolysis of lignocellulosic biomass in water under elevated temperatures and pressures. Journ. superc. Fluid., v. 47, p. 54-63, 2008.

SANTOS, F.; A.; QUEIRÓZ, J.; H.; COLODETTE, J.; L.; FERNANDES, S.; A.; GUIMARÃES, V.; M.; REZENDE, S.; T. Potencial da palha de cana-de-açúcar para a produção de etanol. Quím. Nov., v. 35, p. 1004-1010, 2012.

SANTOS, J.; R.; A.; LUCENA, M.; S.; GUSMÃO, N.; B.; GOUVEIA, E.; R. Optimization of ethanol production by Saccharomyces cerevisiae UFPEDA 1238 in simultaneous saccharification and fermentation of delignified sugarcane bagasse. Ind. Crop. Prod., v. 36, p. 584-588, 2012.

SAVAGE, P. E. Organic chemical reactions in supercritical water. Chem.l Rev., v. 99, p. 603-21, 1999.

SILVA, G. M. Pré-tratamento do bagaço de cana-de-açúcar com amônia aquosa para a produção de etanol. Dissertação de Mestrado. Universidade Federal de São Carlos, Departamento de Engenharia Química, 104 p., 2011. 